\title{
Assessment of the Preserved Biceps Tendon After Arthroscopic Rotator Cuff Repair in Patients $\leq 55$ Years
}

\author{
Itaru Kawashima, M.D., Hiroyuki Sugaya, M.D., Norimasa Takahashi, M.D., \\ Keisuke Matsuki, M.D., Morihito Tokai, M.D., Hideki Hiraiwa, M.D., and \\ Shiro Imagama, M.D.
}

\begin{abstract}
Purpose: We assessed hypertrophy of preserved long head of the biceps tendon (LHBT) and vascularity in the bicipital groove after arthroscopic rotator cuff repair in $\leq 55$-year-old patients and compared postoperative pain between shoulders with or without vascularity in the bicipital groove. Methods: Patients who underwent arthroscopic rotator cuff repair between 2015 and 2017 were reviewed. Inclusion criteria were arthroscopic rotator cuff repair and $\leq 55$ years old. Exclusion criteria were a history of contralateral rotator cuff repair, revision surgery, partial repair or superior capsular reconstruction, shoulder dislocation or fracture, torn LHBT at surgery, LHBT tenodesis, retears, $<1$-year follow-up, and incomplete follow-up data. Cross-sectional area (CSA) of the LHBT and vascularity in the bicipital groove were examined preoperatively and 1 year after surgery using ultrasonography. Shoulder pain at postoperative 1 year was assessed using the pain subscore of the University of California at Los Angeles scale. The data were compared between shoulders with negative and positive vascularity. Results: Fifty-seven shoulders were included in this study. There was no side-to-side difference in preoperative CSA. No difference was found between preoperative and postoperative CSA in the affected shoulders. Postoperative vascularity was identified in 28 (49\%) shoulders. Mean pain score was significantly higher in the negative vascularity group than the positive vascularity group ( 9 and 8 , respectively; $P=.002$ ). Conclusions: The preserved LHBT did not show hypertrophy 1 year after arthroscopic repair of medium-sized or smaller posterosuperior rotator cuff tear in $\leq 55$-year-old patients. However, $49 \%$ of the shoulders postoperatively demonstrated lower-grade vascularity in the bicipital groove. Healthy LHBT can be preserved in $\leq 55$-year-old patients with posterosuperior medium-sized or smaller rotator cuff tears. Level of Evidence: III, retrospective comparative prognostic trial
\end{abstract}

\section{Introduction}

$\mathbf{L}$ ong head of the biceps tendon (LHBT) lesions are considered as a common source of anterior shoulder pain. ${ }^{1}$ They are often associated with rotator cuff tears rather than presented as isolated pathology. ${ }^{2}$ Recently, many surgeons prefer to treat LHBT lesions in addition to rotator cuff repair. ${ }^{3,4}$ We also have been performing biceps tenotomy or tenodesis for most shoulders with rotator cuff tears in middle-aged and

Department of Orthopaedic Surgery, Nagoya University Graduate School of Medicine, Nagoya, Aichi, Japan 466-8550 (I.K., H.H., S.I.); Sports Medicine and Joint Center, Funabashi Orthopaedic Hospital, Funabashi, Chiba, Japan 274-0822 (I.K., N.T., K.M.); and Tokyo Sports $\theta$ Orthopaedic Clinic, Toshima, Tokyo, Japan 170-0012 (H.S., M.T.).

Full ICMJE author disclosure forms are available for this article online, as supplementary material.

Received January 5, 2021; accepted April 27, 2021.

Address correspondence to Itaru Kawashima, M.D., Department of Orthopaedic Surgery, Nagoya University Graduate School of Medicine 65 elderly patients. On the other hand, the higher complication rates after tenotomy have been reported in patients aged $\leq 55$ years. ${ }^{5,6}$ It remains controversial whether the LHBT should be treated in addition to rotator cuff repair in relatively younger patients.

Takahashi et al. ${ }^{7}$ have reported in an ultrasonographic study that the LHBT could be a cause of postoperative pain with progressive hypertrophy of the tendon, even after successful rotator cuff repairs. They

\footnotetext{
Tsurumai, Showa-ku, Nagoya, Aichi 466-8550, Japan. E-mail: itaru. kawashima@gmail.com

(C) 2021 THE AUTHORS. Published by Elsevier Inc. on behalf of the Arthroscopy Association of North America. This is an open access article under the CC BY-NC-ND license (http://creativecommons.org/licenses/by-nc-nd/4.0/). 2666-061X/217

https://doi.org/10.1016/j.asmr.2021.04.006
} 
have also demonstrated that vascularity in the bicipital groove was correlated with postoperative pain. ${ }^{7}$ In addition, significant hypertrophy of the extra-articular LHBT was observed in shoulders with an anterosuperior cuff tear or with a posterosuperior cuff tear larger than medium-sized tear. ${ }^{8}$ It has been reported that patients who underwent rotator cuff repair with biceps tenodesis showed earlier pain relief than patients without LHBT treatment. ${ }^{9}$ On the other hand, it has been reported that LHBT works as a stabilizer of the humeral head ${ }^{10,11}$ and that loss of LHBT was associated with a small increase in superior translation of the humeral head. ${ }^{12}$ According to these findings, we have been preserving the LHBT only when a shoulder with a medium-sized or smaller tear showed no hypertrophy of the extra-articular LHBT and no or mild vascularity in the bicipital groove on preoperative ultrasonography.

However, it was still unknown whether LHBTs that were preserved under our indication would not show progressive hypertrophy after surgery in a relatively young population. Moreover, it was also unclear whether vascularity around preserved LHBTs would be related to postoperative pain.

The purposes of this study were to assess hypertrophy of preserved LHBT and vascularity in the bicipital groove after arthroscopic rotator cuff repair in $\leq 55$ year-old patients and to compare postoperative pain between shoulders with or without vascularity in the bicipital groove. We hypothesized that no significant hypertrophy of the LHBT would be observed after surgery in patients with preserved LHBT and that shoulders with vascularity in the bicipital groove would be related to postoperative pain.

\section{Methods}

\section{Patient Selection}

This retrospective cohort study was approved by the Institutional Review Board and Ethics Committee of Funabashi Orthopaedic Hospital, Funabashi, Chiba, Japan. Medical records of patients who underwent arthroscopic rotator cuff repair at our institute between January 2015 and December 2017 were retrospectively reviewed.

Inclusion criteria of this study were arthroscopic rotator cuff repair and $\leq 55$ years old. Exclusion criteria were history of contralateral rotator cuff repair, revision surgery, partial rotator cuff repair, or superior capsular reconstruction, rotator cuff tears associated with shoulder dislocation or fracture, torn LHBT at surgery, LHBT tenodesis, rotator cuff retear at postoperative 1 -year MRI (Sugaya classification type 4 or $5^{13}$ ), and $<$-year follow up, and incomplete follow-up data.

Patient records, including surgical reports were reviewed for demographic information (age, height, body weight, and body mass index [BMI]) and surgical procedures.

\section{Surgical Procedure and Postoperative Rehabilitation}

Under general anesthesia with an interscalene block, the surgeries were performed in the beach-chair position by multiple surgeons (4 senior surgeons and 3 fellows under senior surgeons' supervision). Diagnostic arthroscopic examination of the glenohumeral joint was conducted via a standard posterior portal with special attention to LHBT lesions. Rotator cuff tear size was determined by each surgeon as mediolateral length, according to DeOrio and Cofield classification: partial; small, $<1 \mathrm{~cm}$; medium, 1 to $3 \mathrm{~cm}$; large, 3 to 5 $\mathrm{cm}$; massive, $>5 \mathrm{~cm}^{14}$ After routine subacromial decompression, rotator cuff tears were repaired using arthroscopic medial knot-tying after suture bridge lateral row repair techniques described by Takeuchi et al. ${ }^{15}$ Partial tears were taken down into full-thickness tears and repaired using the same techniques.

An abduction sling was used for 4 weeks after surgery. On the following day after surgery, isometric rotator cuff exercise and relaxation of the shoulder girdle muscles were initiated. Passive and active-assisted range of motion exercises were initiated after sling removal. Active range of motion and muscle strengthening exercises were initiated 6 weeks after surgery. According to the functional recovery of each patient, light physical activities or exercises were allowed 3 months after surgery, and vigorous physical activities or exercises were allowed 6 months after surgery.

\section{Ultrasonographic Evaluation}

Bilateral shoulders were examined preoperatively and 1 year after surgery by experienced radiologists who were blind to postoperative clinical outcomes of each patient, including pain evaluation using a high-resolution ultrasonography system (EUB7500; Hitachi Medical Corporation, Tokyo Japan). The ultrasound images were obtained with a high-frequency transducer (14 MHz).

To assess the LHBT, the cross-sectional area (CSA) of the LHBT was measured in the bicipital groove using the trapezoidal shape of lesser tuberosity as a reference with the shoulder in a neutral position. ${ }^{7}$ Power Doppler sonography (PDS) was used to evaluate vascularity in the bicipital groove. According to the previous study, ${ }^{7}$ vascularity was evaluated in 4 grades: Grade 0 , no signal or a signal of the anterior circumflex artery; Grade 1 , a single vessel signal other than the anterior circumflex artery; Grade 2, integration of signals that occupied less than half of the image area in the area surrounding the LHBT; and Grade 3, integration of signals that occupied more than half of the image area. In this study, Grade 0 was defined as negative vascularity, and Grade 1 to 3 was defined as positive vascularity. 
Fig 1. Flowchart of patient selection.

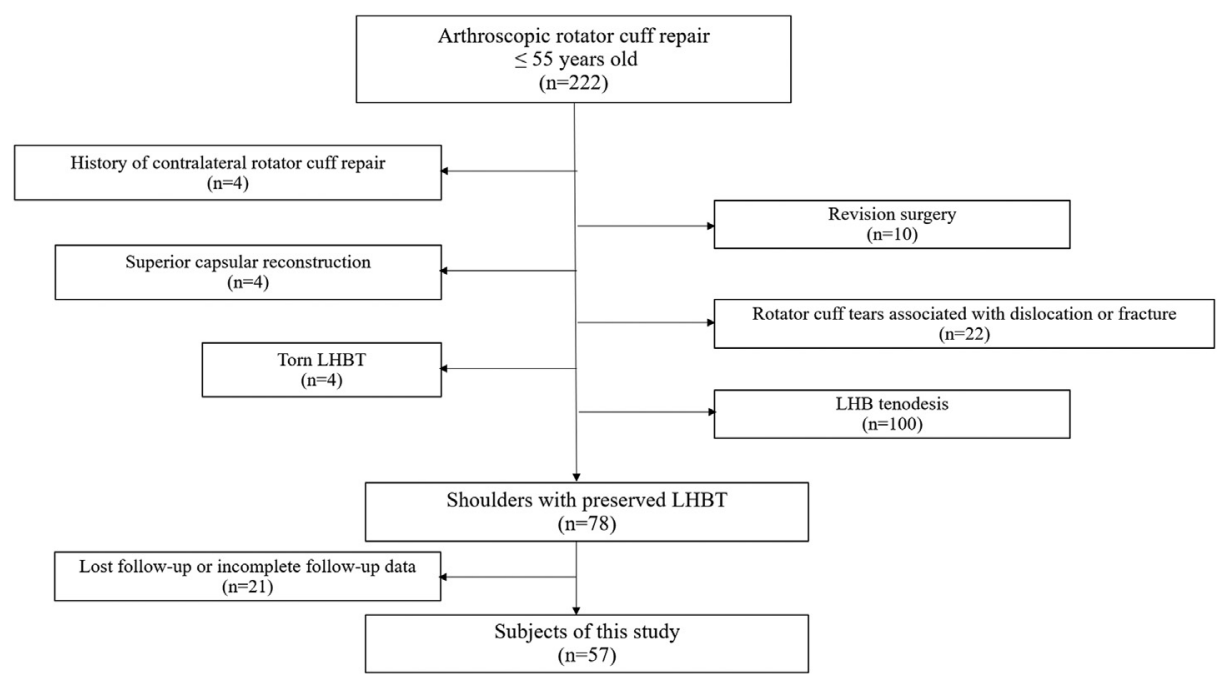

\section{Postoperative Pain Evaluation}

According to the previous study, ${ }^{7}$ shoulder pain at postoperative 1 year was assessed by one of four senior shoulder surgeons (H.S., N.T., K.M., and M.T.) using the pain subscore of the University of California at Los Angeles (UCLA) shoulder rating scale, ${ }^{16}$ which has been reported to have good internal consistency with the Simple Shoulder Test and the Shoulder Pain and Disability Index. ${ }^{17}$ To assess the relationships between postoperative pain and vascularity in the bicipital groove, the shoulders were divided into two groups, according to postoperative vascularity: negative vascularity group (Grade 0 ) and positive vascularity group (Grade 1 to 3 ).

\section{Statistical Analysis}

All statistical analyses were performed using EZR (Saitama Medical Center, Jichi Medical University, Saitama, Japan), which is a graphical user interface for R (The R Foundation for Statistical Computing, Vienna, Austria). More precisely, it is a modified version of $\mathrm{R}$ commander designed to add statistical functions frequently used in biostatistics. The paired $t$-tests and Fisher's exact tests were used to compare preoperative and postoperative outcomes of ultrasonographic evaluation. The Mann-Whitney $U$-tests and Fisher's exact tests were used to compare data between negative and positive vascularity groups. $P$ values $<.05$ were considered statistically significant. A post hoc power analysis was performed on the basis of the data of preoperative and postoperative CSA comparison by use of an $\alpha$ value of .05.

\section{Results}

During the study period, arthroscopic rotator cuff repair was performed in 222 shoulders of 220 patients aged $\leq 55$ years. One-hundred forty-four shoulders of 142 patients were excluded because of a history of contralateral rotator cuff repair (4 shoulders), revision surgery (10 shoulders), superior capsular reconstruction (4 shoulders), rotator cuff tears associated with dislocation or fracture (22 shoulders), torn LHBT at surgery (4 shoulders), and LHB tenodesis (100 shoulders). Among the remaining 78 shoulders, 21 shoulders were excluded due to lost follow-up or incomplete follow-up data. Consequently, this study included 57 shoulders with the follow-up rate of $73 \%$ (57/78) (Fig 1). They consisted of 30 males and 27 females, and the mean age at surgery was 48 years (range: 20-55 years). Rotator cuff tear size was as follows: 33 partial, 19 small, and 5 medium tears (Table 1).

With regards to CSA of the LHBT, there was no difference between the contralateral and affected shoulders before surgery (Table 2; $13 \pm 4 \mathrm{~mm}^{2}$ and $14 \pm 3$ $\mathrm{mm}^{2}$, respectively; $P=.6$ ). There was also no significant differences between preoperative and postoperative CSA of the LHBT in the affected shoulder $\left(14 \pm 3 \mathrm{~mm}^{2}\right.$

Table 1. Demographic Data

\begin{tabular}{lc}
\hline \multicolumn{1}{c}{ Parameter } & Value \\
\hline Male/Female & $30 / 27$ \\
Right/Left & $38 / 19$ \\
Age, year & $48 \pm 7(20-55)$ \\
Height, cm & $165 \pm 9(147-176)$ \\
Body weight, kg & $63.7 \pm 12.3(45.5-9.7)$ \\
BMI, kg/m ${ }^{2}$ & $23.3 \pm 3.5(18.3-31.8)$ \\
Rotator cuff tear size & 33 \\
Partial tear & 19 \\
Small tear & 5 \\
Medium tear & 0 \\
Large tear & 0 \\
Massive tear & 0 \\
Subscapularis tear & \\
\hline The values are given as mean \pm SD (range). \\
BMI, body mass index
\end{tabular}


Table 2. Ultrasonographic Evaluation

\begin{tabular}{lcc}
\hline & Preoperative & Postoperative \\
\hline Contralateral side CSA of LHBT, $\mathrm{mm}^{2}$ & $13 \pm 4(-16-9)$ & \\
CSA of LHBT, $\mathrm{mm}^{2}$ & $14 \pm 3(7-19)^{*}$ & $13 \pm 4(5-21)$ \\
Positive vascularity & $25(44 \%)$ & $28(49 \%)$ \\
& $($ Grade $1: 22,2: 3)$ & $($ Grade $1: 26,2: 2)$ \\
\hline
\end{tabular}

The values are given as means \pm SD (range).

CSA, cross-sectional area; LHBT, long head of the biceps tendon.

*Not significant compared with contralateral side.

and $13 \pm 4 \mathrm{~mm}^{2}$, respectively; $P=.3$ ). The post hoc power analysis indicated that the power of CSA comparison was .7.

There was no difference between preoperative and postoperative vascularity in the bicipital groove (Table 2; preop., 25 of 57 [44\%] shoulders; postop., 28 of 57 [49\%] shoulders; $P=.7$ ). Twenty-nine shoulders with postoperatively negative vascularity and 28 shoulders with positive vascularity were compared (Table 3). Pain subscore of the UCLA scale was significantly higher in the postoperative negative vascularity group than in the postoperative positive vascularity group (Table 3; 9 [range, 6-10] and 8 [range, 1-10], respectively; $P=.002$ ). No significant differences were observed in sex, age, height, body weight, BMI, and rotator cuff tear size between the groups. Moreover, there were no significant differences in preoperative and postoperative CSA of the LHBT and preoperative vascularity in the bicipital groove.

\section{Discussion}

This study demonstrated that no significant hypertrophy was seen in preserved LHBT at 1 year after arthroscopic rotator cuff repair for medium-sized or smaller posterosuperior rotator cuff tears in $\leq 55$-year-old patients with a healthy LHBT. There was no significant difference between preoperative and postoperative CSA of the LHBT; however, $49 \%$ of the shoulders demonstrated postoperative vascularity in the bicipital groove, which was significantly associated with postoperative pain.

Previous reports have shown that CSA of the LHBT in cadaveric shoulders with rotator cuff tears was significantly larger in both the intra-articular and extraarticular portions than those without rotator cuff tears. ${ }^{18,19}$ Desai et al. ${ }^{20}$ reported that about one-third of patients with full-thickness reparable rotator cuff tears are likely to have LHBT pathology. The hypertrophic change of the LHBT has been reported to progress even after successful rotator cuff repairs when not considering patients' age and rotator cuff tear size. ${ }^{7}$ We have been preserving the LHBT when a shoulder with a mediumsized or smaller tear showed no hypertrophy of the extra-articular LHBT, according to findings in previous studies. ${ }^{7,8}$ This study showed that there was no difference between the preoperative and postoperative CSA of the preserved LHBT under our indication in $\leq 55$-yearold patients. Our indications of preserving LHBT were validated at least in younger patients.

Mijic et al. ${ }^{9}$ have reported that patients who underwent rotator cuff repair with biceps tenodesis showed earlier pain relief than patients without LHBT treatment. Watson et al. ${ }^{21}$ have also reported that patients who underwent concomitant biceps tenodesis or

Table 3. Comparison Between Postoperative Negative and Positive Vascularity Groups

\begin{tabular}{|c|c|c|c|}
\hline & Negative Vascularity $(n=29)$ & Positive Vascularity $(n=28)$ & $P$ Value \\
\hline Male/Female & $15 / 14$ & $15 / 13$ & 1 \\
\hline Right/Left & $19 / 10$ & $19 / 9$ & 1 \\
\hline Age, yr & $48 \pm 5(33-55)$ & $48 \pm 8(20-55)$ & .5 \\
\hline Body weight, kg & $64.2 \pm 12.6(46.9-85.4)$ & $63.3 \pm 12.3(47.0-72.0)$ & .9 \\
\hline $\mathrm{BMI}, \mathrm{kg} / \mathrm{m}^{2}$ & $23.6 \pm 4.0(18.3-31.4)$ & $23.1 \pm 2.9(19.1-31.8)$ & 1.0 \\
\hline Rotator cuff tear size (partial/small/medium) & $18 / 10 / 1$ & $15 / 9 / 4$ & .4 \\
\hline Preoperative side-to-side difference of CSA, $\mathrm{mm}^{2}$ & $0 \pm 5(-16-9)$ & $1 \pm 4(-7-10)$ & .5 \\
\hline Preoperative CSA of affected side, $\mathrm{mm}^{2}$ & $14 \pm 4(7-19)$ & $13 \pm 3(8-19)$ & .4 \\
\hline Postoperative CSA of affected side, $\mathrm{mm}^{2}$ & $13 \pm 4(5-21)$ & $13 \pm 3(6-19)$ & .8 \\
\hline \multirow{2}{*}{ Preoperative vascularity } & $10(34 \%)$ & $15(54 \%)$ & .2 \\
\hline & (Grade $1: 9,2: 1$ ) & (Grade $1: 13,2: 2$ ) & \\
\hline
\end{tabular}

The values are given as means \pm SD (range). Bolded value indicates significant difference.

CSA, cross-sectional area; LHBT, long head of the biceps tendon. 
tenotomy at the time of rotator cuff repair demonstrated greater improvement at postoperative 1 year compared with isolated rotator cuff repair patients. In this study, residual vascularity in the bicipital groove was seen in almost half of the shoulders one year after surgery, and the vascularity was significantly associated with pain. However, the vascularity was Grade 1 in most shoulders. In addition, the mean pain score was 9 and 8 out of 10 points in the negative and positive vascularity groups, respectively, which meant that pain was mild in both groups and that the difference was probably subclinical, considering that the reported minimal clinically important difference of UCLA scale was 8.7. ${ }^{22}$ The present study might also indicate the diagnostic usefulness of ultrasonography to detect the origin of minor pain by revealing low-grade vascularity in the bicipital groove after successful rotator cuff repair.

This study suggested that healthy LHBT in $\leq 55$-yearold patients could be preserved when rotator cuff tears were posterosuperior medium-sized or smaller tears. However, careful assessment may be required after rotator cuff repair without biceps treatment, especially assessment for vascularity in the bicipital groove. Ultrasonography is useful to assess vascularity around the LHBT due to its noninvasiveness and practicality.

\section{Limitations}

This study had several limitations. First, this was a retrospective study with one-year follow-up. The outcomes of this study might be more clinically relevant with a longer follow-up period. Second, concomitant procedures such as rotator cuff repair may have some effect on the outcomes. Third, the UCLA pain score is not specific to biceps-related pain. Fourth, surgeries were done by multiple surgeons. Although we were following our surgical policy, there might be a slight difference in surgical indications and procedures. Fifth, $27 \%(21 / 78)$ of patients were lost to follow-up. Sixth, the study was underpowered with a power of .7. Lastly, the sample size was relatively small.

\section{Conclusions}

The preserved LHBT did not show hypertrophy 1 year after arthroscopic repair of medium-sized or smaller posterosuperior rotator cuff tear in $\leq 55$-year-old patients. However, $49 \%$ of the shoulders postoperatively demonstrated lower-grade vascularity in the bicipital groove. Healthy LHBT can be preserved in $\leq 55$-yearold patients with posterosuperior medium-sized or smaller rotator cuff tears.

\section{Acknowledgment}

We are grateful to Koharu Matsuki for English language editing.

\section{References}

1. Hsu AR, Ghodadra NS, Provencher MT, et al. Biceps tenotomy versus tenodesis: a review of clinical outcomes and biomechanical results. J Shoulder Elbow Surg 201 1;20: 326-332.

2. Nho SJ, Shindle MK, Sherman SL, et al. Systematic review of arthroscopic rotator cuff repair and mini-open rotator cuff repair. J Bone Joint Surg Am 2007;89: $127-136$.

3. Kim SH, Yoo JC. Arthroscopic biceps tenodesis using interference screw: End-tunnel technique. Arthroscopy 2005;21:1405.

4. Koh KH, Ahn JH, Kim SM, et al. Treatment of biceps tendon lesions in the setting of rotator cuff tears: prospective cohort study of tenotomy versus tenodesis. Am J Sports Med 2010;38:1584-1159.

5. Mirzayan R, McCrum C, Butler KR, et al. Risk factors and complications following arthroscopic tenotomy of the long head of the biceps tendon. Orthop J Sports Med 2020;8:2325967120904361.

6. Chen RE, Voloshin I. Long head of biceps injury: treatment options and decision making. Sports Med Arthrosc Rev 2018;26:139-144.

7. Takahashi N, Sugaya H, Matsumoto M, et al. Progression of degenerative changes of the biceps tendon after successful rotator cuff repair. J Shoulder Elbow Surg 2016;26: 424-429.

8. Takahashi N, Sugaya H, Matsuki K, et al. Hypertrophy of the extra-articular tendon of the long head of biceps correlates with the location and size of a rotator cuff tear. Bone Joint J 2017;99-B:806-811.

9. Mijic D, Kurowicki J, Berglund D, et al. Effect of biceps tenodesis on speed of recovery after arthroscopic rotator cuff repair. JSES Int 2020;4:341-347.

10. Itoi E, Motzkin EN, Morrey FB, et al. Stabilizing function of the long head of the biceps in the hanging arm position. J Shoulder Elbow Surg 1994;3:135-142.

11. Eshuis R, Gast DA. Role of the long head of the biceps brachii muscle in axial humeral rotation control. Clin Anat 2012;25:737-745.

12. Warner JJ, McMahon JP. The role of the long head of the biceps brachii in superior stability of the glenohumeral joint. J Bone Joint Surg Am 1995;77: 366-372.

13. Sugaya H, Maeda K, Matsuki K, et al. Repair integrity and functional outcome after arthroscopic double-row rotator cuff repair. A prospective outcome study. J Bone Joint Surg Am 2007:89:953-996.

14. DeOrio JK, Cofield RH. Results of a second attempt at surgical repair of a failed initial rotator-cuff repair. J Bone Joint Surg Am 1984;66:563-567.

15. Takeuchi Y, Sugaya H, Takahashi N, et al. Repair integrity and re-tear pattern after arthroscopic medial knot-tying after suture bridge lateral row rotator cuff repair. Am J Sports Med 2020;48:2510-2517.

16. Ellman H, Hanker G, Bayer M. Repair of the rotator cuff. End-result study of factors influencing reconstruction. J Bone Joint Surg Am 1986;68:1136-1144.

17. Roddey TS, Olson SL, Cook KF, et al. Comparison of the University of California-Los Angeles Shoulder Scale and 
the Simple Shoulder Test with the Shoulder Pain and Disability Index: Single-administration reliability and validity. Phys Ther 2000;80:759-768.

18. Aizawa T, Itoi E, Minagawa H, et al. Cross-sectional area of the tendon and the muscle of the biceps brachii in shoulders with rotator cuff tears: a study of 14 cadaveric shoulders. Acta Orthop 2005;76: 509-512.

19. Sakurai G, Ozaki J, Tomita Y, et al. Morphologic changes in long head of biceps brachii in rotator cuff dysfunction. J Orthop Sci 1998;3:137-142.
20. Desai SS, Mata KH. Long head of biceps tendon pathology and results of tenotomy in full-thickness reparable rotator cuff tear. Arthroscopy 2017;33:1971-1976.

21. Watson ST, Robbins CB, Bedi A, et al. Comparison of outcomes 1 year after rotator cuff repair with and without concomitant biceps surgery. Arthroscopy 2017;33: 1928-1936.

22. Simovitch R, Flurin PH, Wright T, et al. Quantifying success after total shoulder arthroplasty: the minimal clinically important difference. J Shoulder Elbow Surg 2018;27: 298-305. 